\title{
Comparação de habilidades cognitivas de crianças a termo e pré-termo
}

\section{Comparison of cognitive skills of children to term and pre-term}

\section{Comparación de habilidades cognitivas de niños a término y pre-término}

\author{
Laís Galvão Moura Tenório* \\ Cristiane Monteiro Pedruzzi* \\ Alexsandra Silva Santos* \\ Antonio Roque de Lima dos Santos*
}

\section{Resumo}

Introdução: A cognição é um conjunto de habilidades mentais essenciais para se adquirir conhecimento. É durante os três primeiros anos de vida que ocorrem os grandes avanços no desenvolvimento cognitivo. Este desenvolvimento está diretamente ligado aos aspectos nutricionais, socioeconômicos, nível educacional da família e à dinâmica familiar. Crianças pré-termo têm risco para alteração no processo do desenvolvimento cognitivo. Objetivo: Verificar o desempenho cognitivo em 20 crianças pré-termo. Método: É um estudo observacional e transversal. A amostra foi composta por 20 crianças nascidas a termo e 20 nascidas pré-termo. Os responsáveis pelas crianças responderam a um questionário contendo informações referentes à história clínica do participante e dados socieconômicos. Foi aplicada a subparte da escala Bayley III referente às habilidades cognitivas. A análise dos dados foi realizada pelo teste estatístico de Mann-Whitney, apresentados em dados quantitativos de médias, desvio padrão e alfa (p-valor). Resultados: Ao analisar as pontuações das habilidades cognitivas dos grupos observou-se que 16 crianças ( $80 \%$ ) encontravam-se com pontuações abaixo da média, $1(5 \%)$ na média e $3(15 \%)$ acima da média esperada para a idade. O grupo pré-termo obteve 13 crianças $(65 \%)$ com pontuações abaixo da média, $1(5 \%)$ na média e $6(30 \%)$ acima da média, notando-se que o grupo prétermo apresentou melhores resultados. Não houve diferença significativa entre as médias dos dois grupos

* Universidade Estadual de Ciências da Saúde de Alagoas, Maceió, Alagoas, Brasil

Contribuição dos autores:

LGM: Elaboração de artigo e coleta de dados; CMP: Elaboração de projeto e artigo; ASS: Coleta de dados e contribuições na revisão do artigo; ARLS: Coleta de dados.

E-mail para correspondência: Laís Galvão Moura Tenório lais_gmtl@hotmail.com Recebido: $27 / 11 / 2017$

Aprovado:29/10/2018 
do presente estudo em relação a nenhuma das variáveis. Conclusão: Não houve diferença estatística no desempenho cognitivo entre os grupos nesta faixa etária. Deve-se considerar que os desempenhos dos participantes poderão mudar em outra faixa etária.

Palavras-chave: Cognição; Prematuridade; Escala Bayley III; Desenvolvimento Infantil.

\section{Abstract}

Introduction: Cognition is a set of mental skills essential to acquiring knowledge. It is during the first three years of life that great advances in cognitive development occur. This development is directly related to the nutritional, socioeconomic aspects, educational level of the family and family dynamics. Premature children are at risk for a change in the cognitive development process. Objective: To verify cognitive development in 20 preterm infants. Method: It is an observational and cross-sectional study. The sample consisted of 20 full-term infants and 20 preterm infants. Those responsible for the children answered a questionnaire, containing information regarding the participant's medical history and socioeconomic data. It was applied to the subpart of the Bayley III scale regarding cognitive abilities. Data analysis was performed using the Mann-Whitney statistical test, presented in quantitative data of means, standard deviation and alpha (p-value). Results: When analyzing the scores of the cognitive abilities of the groups it was observed that 16 children ( $80 \%)$ had 2 below-average scores, $1(5 \%)$ on average and $3(15 \%)$ above the expected age-for-age. The preterm group obtained 13 children $(65 \%)$ with below-average scores, $1(5 \%)$ in the mean and $6(30 \%)$ above the mean, noting that the premature group presented better results. There was no significant difference between the means of the two groups of the present study in relation to none of the variables. Conclusion: There was no statistical difference in cognitive performance between the groups in this age group. It should be considered that the performance of the participants may change in another age group.

Keywords: Cognition; Prematurity; Bayley III Scale; Child Development.

\section{Resumen}

Introducción: La cognición es un conjunto de habilidades mentales esenciales para adquirir conocimiento. Es durante los tres primeros años de vida que ocurren los grandes avances en el desarrollo cognitivo. Este desarrollo está directamente ligado a los aspectos nutricionales, socioeconómicos, nivel educativo de la familia y la estimulación favorecida en la relación familiar. Los niños prematuros tienen valor predictivo para cambiar el proceso en el desarrollo cognitivo. Objetivo: Verificar el desarrollo cognitivo en 20 niños prematuros. Método: Es un estudio observacional y transversal. La muestra fue compuesta por 20 niños nacidos a término y 20 nacidos de pre-término. Los responsables de los niños respondieron a un cuestionario, conteniendo informaciones referentes a la historia clínica del participante y datos socieconómicos. Se aplica la prueba equivalente a las habilidades cognitivas de la escala Bayley III. El análisis de los datos fue realizado por los test estadísticos de Mann-Whitney, presentados en datos cuantitativos de promedios, desviación estándar y alfa (p-valor). Resultados: $\mathrm{Al}$ analizar las puntuaciones de las habilidades cognitivas de los grupos se observó que 16 niños (80\%) se encontraban con puntuaciones por debajo de la media, $1(5 \%)$ en la media y $3(15 \%)$ por encima de la media esperada para la edad. El grupo anterior cuenta con 13 niños $(65 \%)$ con puntuaciones por debajo de la media, $1(5 \%)$ en la media y $6(30 \%)$ por encima de la media, notando que el grupo prematuro presentó mejores resultados. En el diferencial diferencia significativa entre las medias de los de los grupos del presente estudio en relación a varias de las variables. Conclusión: En el intervalo diferencia estadística en el ritmo cognitivo de los de los grupos en este grupo de edad. Se debe considerar que estos resultados pueden cambiar en otro grupo de edad infantil.

Palabras claves: Cognición; Prematuridad; Escala Bayley III; Desarrollo Infantil. 


\section{Introdução}

A cognição e a linguagem são interdependentes; por esta razão, uma influenciará a outra. Pode-se definir cognição como um conjunto de habilidades mentais essenciais para adquirir conhecimento. Essas habilidades envolvem principalmente o pensamento, raciocínio, abstração, memória, atenção, criatividade e resolução de problemas. Em geral, as habilidades cognitivas proporcionam ao sujeito uma reflexão acerca das informações e ações no seu cotidiano ${ }^{1}$. Os grandes avanços no desenvolvimento cognitivo, motor, social e na linguagem ocorrem durante os três primeiros anos de vida e são indispensáveis para o desempenho global $^{2}$.

Os aspectos nutricionais, socioeconômicos, nível educacional da família e a estimulação favorecida na relação familiar estão diretamente ligados ao desenvolvimento global da criança. Quando algum desses aspectos influencia de forma negativa, tem-se o risco de atraso e/ou distúrbio no desenvolvimento infantil ${ }^{2}$. Da mesma maneira, a prematuridade e o baixo peso ao nascer são alguns dos principais fatores de risco para desencadear alguma alteração no desenvolvimento ${ }^{3}$.

O recém-nascido pré-termo (RNPT) é aquela criança que tem idade gestacional inferior a 37 semanas ${ }^{3}$. Os RNPT têm sido identificados com menores habilidades de cognição e memória. Além do fator prematuridade, os riscos sociais estão interligados ao desenvolvimento, e a soma de diversos fatores afeta potencialmente o desenvolvimento cognitivo ${ }^{4}$.

Estudos longitudinais mostram que entre $30 \mathrm{e}$ $50 \%$ dos RNPT apresentam dificuldades cognitivas que podem ser detectadas em idade pré-escolar. Por esta razão, é de suma importância investigar o desenvolvimento cognitivo em idade precoce, identificando as crianças com risco para alteração, possibilitando a intervenção prévia e orientando os pais quanto às dificuldades e os possíveis tratamentos ${ }^{5}$.

A estimulação precoce é uma prioridade determinante, pois poderá proporcionar qualidade de vida não apenas para a criança e sua família, mas influencia o planejamento financeiro das políticas públicas para toda a sociedade. Destaca-se a estimulação das funções executivas, as quais estão diretamente relacionadas à cognição, como a memória de trabalho, controle dos impulsos, capacidade de planejamento e atenção. Essas habilidades não são inatas, entretanto, nascemos com o potencial para aprendê-las. Tal aprendizado inicia-se ao nascimento, desenvolvendo-se através de nossas experiências adquiridas ${ }^{6}$.

As funções executivas são essenciais tanto para aquisições cognitivas como para as sociais e emocionais. Essas habilidades tornam-se mais evidentes a partir dos três anos de idade, quando a criança é capaz de seguir comando de duas etapas, realizar escolhas simples, manter a atenção e recontar. Entretanto, é possível vê-las de forma mais sutil ao final do primeiro ano de vida ${ }^{6}$.

Dentre as escalas avaliativas consideradas padrão-ouro, está a Bayley III, desenvolvida por Nancy Bayley e colaboradores em 2006, e que tem sido usada em pesquisas científicas para a avaliação do desenvolvimento infantil. Avaliam-se cinco áreas do desenvolvimento infantil: cognitiva, de linguagem (receptiva e expressiva), motor (fino e grosso), socioemocional e comportamento adaptativo. É um instrumento de referência utilizado em muitos países, entretanto, ainda não foi validado no Brasil, sendo utilizado apenas para fins científicos ${ }^{7,8}$

A partir do pressuposto de que a prematuridade é um fator que pode gerar alterações no desenvolvimento cognitivo, o presente estudo tem como objetivo verificar o desenvolvimento cognitivo em crianças PT.

\section{Métodos}

Esta é uma pesquisa observacional e transversal, a qual foi submetida e aprovada pelo Comitê de Ética em Pesquisa, sob o registro 48561115.0.0000.5011. O levantamento de crianças na faixa etária entre 24 a 30 meses foi realizado em uma maternidade escola do Estado de Alagoas. A partir desse grupo foi selecionada a amostragem para o estudo, por meio de convites individuais aos responsáveis pelos menores. Os que aceitaram, assinaram um Termo de Consentimento Livre e Esclarecido (TCLE) e o Termo de Assentimento.

Nesta pesquisa foram incluídos 40 participantes que não realizavam terapia fonoaudiológica até o momento de coleta dos dados. A amostra é constituída por 20 crianças nascidas a termo (AT) e 20 pré-termo (PT), de ambos os sexos, assim como sem alterações visuais, auditivas, neurológicas e/ 
ou algum grau do transtorno do espectro autista (TEA) diagnosticado.

Os dados dos participantes, tais como história clínica, dados socioeconômicos, estatura e peso, foram considerados para posterior análise. Além desses dados, a subparte da Bayley Scales of Infant Development - BSID-III, ou simplesmente Bayley III, correspondente ao domínio cognitivo, foi aplicada. O ponto inicial do teste foi equivalente à idade cronológica da criança, sendo finalizado após cinco erros consecutivos. A execução das etapas da escala foram pontuadas, com 0 para não realização e 1 para o desempenho apropriado da prova proposta, conforme o manual. Os aplicadores da escala foram estagiários do curso de Fonoaudiologia, sendo estes supervisionados por uma Fonoaudióloga e Psicóloga.

As tarefas propostas na subparte cognitiva envolvem apresentar um ou mais objetos para a criança de maneira individual. Esses objetos servem para a realização de uma atividade, por exemplo, pinos - estilo bastão de lápis de cera - para encaixar em uma prancha com pequenos orifícios. O teste foi aplicado em uma ou, no máximo, duas visitas, com duração, aproximadamente, de uma hora para cada visita. O dia, local e horário mais convenientes para os participantes foram agendados para a realização da coleta.

As pontuações obtidas na Bayley III foram registradas em formulários para uma posterior análise (Figura 1). A contabilidade de acertos foi convertida em escores, determinando se o participante obteve pontuação na BSID-III acima da média, na média ou abaixo da média, de acordo com sua idade. Estas pontuações foram comparadas entre os dois grupos, assim como as outras variáveis, sendo estas: estado nutricional (peso-altura e peso-idade), escolaridade do responsável e renda familiar mensal.

Os dados coletados foram tabulados no software Excel Office 2010 e posteriormente analisados através do programa para apuração estatística BioEstat 5.3, sendo realizada a estatística descritiva dos dados (média, mediana e desvio padrão). Além disso, foi aplicado o teste Mann-Whitney para comparar as crianças PT com as nascidas AT, em relação ao desenvolvimento cognitivo. $\mathrm{O}$ p-valor (alfa) considerado foi 0,05 .

\section{Resultados}

Ao analisar as pontuações das habilidades cognitivas dos dois grupos, observou-se média do grupo AT de 2.65 (desvio padrão de 0.74 ), estando 16 crianças $(80 \%)$ com pontuações abaixo da média, $1(5 \%)$ na média e $3(15 \%)$ acima da média esperada para a idade. Contudo, o grupo PT obteve uma média de 2.35 (desvio padrão de 0.93 ) com 13 crianças $(65 \%)$ com pontuações abaixo da média, $1(5 \%)$ na média e $6(30 \%)$ acima da média, notando-se que o grupo PT apresentou mais crianças acima da média. Entretanto não houve diferença significativa entre as médias dos dois grupos do presente estudo. (Tabelas 1 e 2)

O grupo AT apresentou, em relação ao estado nutricional peso/estatura, uma média de 4.2 (desvio padrão de 1.07), 2 (10\%) das 20 crianças apresentaram obesidade, 7 (35\%) sobrepeso, 6 (30\%) estavam com risco de sobrepeso, 4 (20\%) eutróficas e apenas $1(5 \%)$ criança com magreza. O grupo PT apresentou uma média de 4.20 (desvio padrão de 1.11$)$, no qual 3 (15\%) crianças apresentaram obesidade, 5 (25\%) sobrepeso, 5 (25\%) estavam com risco de sobrepeso e 7 (35\%) eutróficas, não havendo nenhuma criança apresentando magreza (Tabela 1).

Quando observado o estado nutricional em relação ao peso/idade das crianças AT, houve uma média de 2.95 (desvio padrão 0.51 ), sendo 1 (5\%) com peso elevado para idade, 18 (90\%) com peso adequado para idade e $1(5 \%)$ apresentando muito baixo peso para a idade. O grupo PT obteve média de 3.05 (desvio padrão de 0.22 ), com 1 ( $5 \%$ ) criança apresentando peso elevado para idade e 19 (95\%) com peso adequado para a idade (Tabela 1).

Em relação à escolaridade de responsáveis, observou-se como resultado uma média de 10.45 das mães do grupo AT, já o grupo PT obteve média de 10.20. Vale ressaltar que $4(20 \%)$ responsáveis do grupo PT estudaram por 17 anos e outras $11(55 \%)$ por menos de 12 anos. Já no grupo AT, apenas 2 (10\%) responsáveis estudaram por 17 anos, entretanto, apenas 9 (45\%) estudaram por menos de 12 anos. A renda familiar das crianças AT apresentou média de R\$1034.20 (desvio padrão de 737.66) e do grupo PT média de R $\$ 1324.7$ (desvio padrão de 865.0), não havendo uma diferença significativa entre os dois grupos (Tabela 1). 


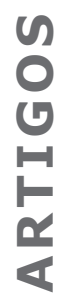

\section{Screening Test Record Form}

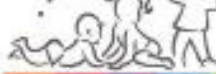

Bayley

Child's name

Scales of Infant and

Toddler Development"

Sex: $\square M$ D F ID :

THIRDEDITION

Examiner's name:

Schoolichild care program:

Reason for referral:

TEST

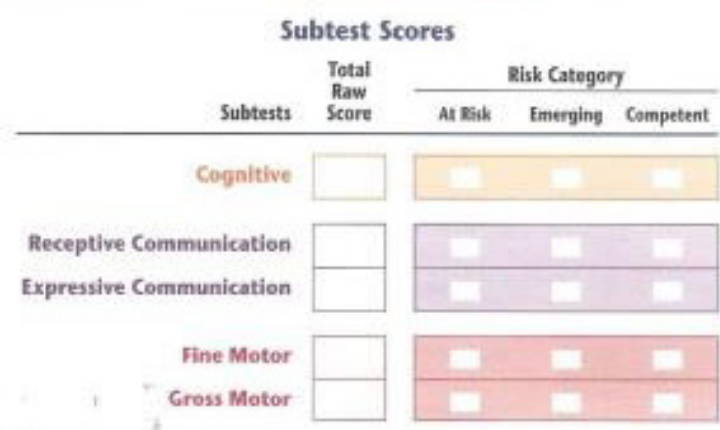

Comments

\begin{tabular}{|c|c|c|c|}
\hline \multicolumn{4}{|c|}{ Calculate Age } \\
\hline & Yearn & Months & Days \\
\hline \multicolumn{4}{|l|}{ Duse Teited } \\
\hline \multicolumn{4}{|l|}{ Date of Eirth } \\
\hline \multicolumn{4}{|l|}{ Age } \\
\hline \multicolumn{4}{|l|}{$\begin{array}{l}\text { Age in Months } \\
\text { and Days }\end{array}$} \\
\hline $\begin{array}{l}\text { Adjustment fox } \\
\text { Premaburty }\end{array}$ & $\begin{array}{l}\text { Mejuet itrough } \\
\text { 24 menthi }\end{array}$ & & \\
\hline \multicolumn{4}{|l|}{ Adjusted $/ g$ : } \\
\hline 5tart Point & \multicolumn{2}{|c|}{$\begin{array}{l}\text { Cituine ifart point } \\
\text { arcending 10 durt bolow }\end{array}$} & \\
\hline \multicolumn{3}{|c|}{ Age* } & $\begin{array}{l}\text { Start } \\
\text { Polnt }\end{array}$ \\
\hline 1-6 months & & & A \\
\hline $7-12$ months & & & B \\
\hline 13-24 months & & & C \\
\hline $25-42$ months & & & D \\
\hline
\end{tabular}

-Round child's age to the nearest month.

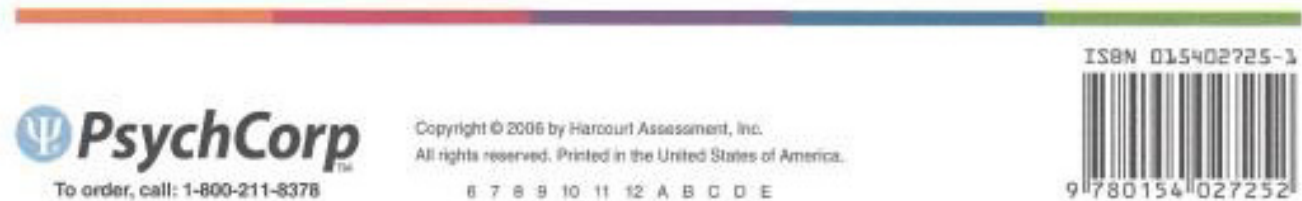

Figura 1. 
Reversal Rule: The child must obtain a score of 1 on the first item at the start point of any age to go forward. If the child obtains a score of zero on the first item, go back to the start point for the previous age and administer those items.

Discontinue Rule: Stop administration when the child obtains scores of zero on four consecutive items.

\begin{tabular}{|c|c|c|c|}
\hline द्2 Item & Materials & Score Criteria and Comments & Score \\
\hline \multirow{2}{*}{$\begin{array}{l}\text { 1. Regards Object } \\
\text { for } 3 \text { Seconds }\end{array}$} & \multirow{2}{*}{$\begin{array}{l}\text { Ring with string. } \\
\text { ball, or otber } \\
\text { small object of } \\
\text { interest }\end{array}$} & Score: Child gazes contimabusly at abject lor at lesst 3 seconds. & \multirow[b]{2}{*}{10} \\
\hline & & & \\
\hline \multirow{2}{*}{$\begin{array}{l}\text { 2. Recognizes } \\
\text { Caregiver }\end{array}$} & \multirow[t]{2}{*}{ Nocie } & Score: Child's expression changes to indicate recognition of the caregiver. & \multirow[b]{2}{*}{10} \\
\hline & & & \\
\hline \multirow{2}{*}{$\begin{array}{l}\text { 3. Becomes Excited } \\
\text { in Anticipation }\end{array}$} & \multirow[t]{2}{*}{ None } & Score: Child displays anticipatory excitement: & \multirow[b]{2}{*}{10} \\
\hline & & & \\
\hline \multirow{2}{*}{$\begin{array}{l}\text { 4. Regards Object } \\
\text { for } 5 \text { Seconds }\end{array}$} & \multirow{2}{*}{$\begin{array}{l}\text { Block or other } \\
\text { small object of } \\
\text { interest } \\
\text { Stopwatch } \\
\text { \& } 5 \text { seconds }\end{array}$} & Score: Child regards object continuously for at least 5 seconds. & \multirow[b]{2}{*}{10} \\
\hline & & & \\
\hline \multirow{2}{*}{$\begin{array}{l}\text { 5. Habituates } \\
\text { to Picture } \\
\text { (Balloons) }\end{array}$} & \multirow{2}{*}{$\begin{array}{l}\text { Stimulus Book } \\
(p .5) \\
\text { Stopwatch } \\
030 \text { seconds }\end{array}$} & $\begin{array}{l}\text { Score: Child habituates within } 30 \text { seconds, displaying decrease in attention } \\
\text { and interest. }\end{array}$ & \multirow[b]{2}{*}{$1<$} \\
\hline & & & \\
\hline \multirow{2}{*}{$\begin{array}{l}\text { 6. Prefers Novel } \\
\text { Picture (Bail) }\end{array}$} & \multirow{2}{*}{$\begin{array}{l}\text { Stimulus Book } \\
\text { (pp. 7-9) } \\
\text { Stopwatch } \\
\$ 15 \text { seconds } \\
\text { per page }\end{array}$} & Score: Child looks longer at ball than balloons in both presentations. & \multirow[b]{2}{*}{10} \\
\hline & & & \\
\hline \multirow{2}{*}{$\begin{array}{l}\text { 7. Responds } \\
\text { to Navel } \\
\text { Surroundings }\end{array}$} & \multirow[t]{2}{*}{ None } & $\begin{array}{l}\text { Score: Child displays awareness of being in novel surroundings } \\
\text { (e.g., startles, locks around) }\end{array}$ & \multirow[b]{2}{*}{10} \\
\hline & & & \\
\hline \multirow[t]{2}{*}{$\begin{array}{l}\text { 8. Persistent } \\
\text { Reaxh }\end{array}$} & \multirow{2}{*}{$\begin{array}{l}\text { Block without } \\
\text { hole or other } \\
\text { small object of } \\
\text { interest }\end{array}$} & $\begin{array}{l}\text { Score: Child persistently reaches for object, even if he or she falls } \\
\text { to obtain it. }\end{array}$ & \multirow[b]{2}{*}{10} \\
\hline & & & \\
\hline \multirow{2}{*}{$\begin{array}{l}\text { 9. Pulls String } \\
\text { Adaptively }\end{array}$} & \multirow[t]{2}{*}{ Ring with string } & Score: Child picks up string, purposely pulls to secure ring, and grasps ring. & \multirow[b]{2}{*}{1} \\
\hline & & & \\
\hline \multirow{2}{*}{$\begin{array}{l}\text { 10. Retains Both } \\
\text { Blocks }\end{array}$} & \multirow{2}{*}{$\begin{array}{l}2 \text { blocks without } \\
\text { hales }\end{array}$} & Score: Child holds both blocks simultaneously for at least 3 seconds. & \multirow[b]{2}{*}{10} \\
\hline & & & \\
\hline
\end{tabular}

Figura 1. Modelo da folha de rosto e das atividades da Escala Bayley III 
Tabela 1. Dados descritivos em média e desvio padrão das variáveis sociais e nutricionais dos grupos pré-termo e a termo

\begin{tabular}{|c|c|c|c|c|}
\hline Variáveis & Crianças & Média & $\begin{array}{l}\text { Desvio } \\
\text { padrão }\end{array}$ & Mediana \\
\hline \multirow{2}{*}{ Estado nutricional (peso-estatura) } & A termo & 4.25 & 1.07 & 4.0 \\
\hline & Pré-termo & 4.20 & 1.11 & 4.0 \\
\hline \multirow[t]{2}{*}{ Estado nutricional (peso-idade) } & A termo & 2.95 & 0.51 & 3.0 \\
\hline & Pré-termo & 3.05 & 0.22 & 3.0 \\
\hline \multirow[t]{2}{*}{ Escolaridade do responsável (anos) } & A termo & 10.45 & 3.64 & 12.0 \\
\hline & Pré-termo & 10.20 & 4.45 & 9.0 \\
\hline \multirow[t]{2}{*}{ Renda familiar(R\$) } & A termo & 1034.20 & 737.66 & 865.0 \\
\hline & Pré-termo & 1324.7 & 1211.71 & 930.0 \\
\hline \multirow[t]{2}{*}{ Pontuação da escala } & A termo & 2.65 & 0.74 & 3.0 \\
\hline & Pré-termo & 2.35 & 0.93 & 3.0 \\
\hline
\end{tabular}

Tabela 2. Pontuações das crianças na escala BSID-III

\begin{tabular}{ccc}
\hline Variáveis & $\begin{array}{c}\mathbf{N}^{\mathbf{0}} \text { de } \\
\text { A Termo }\end{array}$ & $\begin{array}{c}\mathbf{N}^{\mathbf{0}} \mathbf{\text { de }} \\
\text { Pré Termo }\end{array}$ \\
\hline Pontuação acima da média & 3 & 6 \\
Pontuação na média & 1 & 1 \\
Pontuação abaixo da média & 16 & 13 \\
\hline
\end{tabular}

Através da análise inferencial estatística destes escores, pelo teste de Mann Whitney, obtidos pelo desempenho nas habilidades cognitivas entre as crianças AT e PT, observou-se que os dois grupos não apresentaram diferença estatisticamente relevante, apresentando p-valor de 0.3942 em relação às pontuações (Tabela 3 ).
A mesma correlação estatística foi realizada entre as outras variáveis, comparando sempre os dois grupos do estudo. Não se observou nenhuma evidência estatística significativa de $\mathrm{p}<0,05$ entre as variáveis analisadas (Tabela 3).

Tabela 3. Correlações estatísticas entre as variáveis sociais, nutricionais e de habilidades cognitivas dos grupos pré-termo e a termo

\begin{tabular}{|c|c|c|}
\hline Variáveis & Crianças & p-valor \\
\hline Estado nutricional (peso-estatura) & $\begin{array}{l}\text { A termo } \\
\text { Pré-termo }\end{array}$ & 0,7972 \\
\hline Estado nutricional (peso-idade) & $\begin{array}{c}\text { A termo } \\
\text { Pré-termo }\end{array}$ & 0,7972 \\
\hline Escolaridade Materna (anos) & $\begin{array}{l}\text { A termo } \\
\text { Pré-termo }\end{array}$ & 0,6652 \\
\hline Renda $(\mathrm{R} \$)$ & $\begin{array}{c}\text { A termo } \\
\text { Pré-termo }\end{array}$ & 0.4989 \\
\hline Pontuação da escala & $\begin{array}{l}\text { A termo } \\
\text { Pré-termo }\end{array}$ & 0,3942 \\
\hline
\end{tabular}

Legenda: P-valor calculado a partir do teste Mann Whitney 


\section{Discussão}

Neste estudo, o grupo AT obteve mais pontuações abaixo da média (80\%) do que o grupo PT $(65 \%)$. Apesar disso, os resultados não apresentaram relevância significativa. Esses resultados não são determinantes, isto é, algumas dificuldades cognitivas podem aparecer com o aumento da idade cronológica. A maioria dos déficits ou distúrbios cognitivos e comportamentais podem ser mais bem observados durante a idade escolar ${ }^{9}$. Esse achado justifica o fato de não haver resultados significativos no presente estudo quando comparados os dois grupos, pois estes encontravam-se em idade pré-escolar.

Ademais, observou-se que as crianças PT apresentaram resultados equivalentes aos das crianças AT em relação a todas as variáveis, assim como os escores da avaliação cognitiva realizada através da BSID-III, como já citado anteriormente. De acordo com a literatura, o fator social pode influenciar o desenvolvimento mais do que os fatores biológicos; entretanto, com o avanço da idade, o impacto dos fatores biológicos precoces, tais como o nascimento pré-termo, aumenta, caso não ocorra nenhum cuidado específico, o que pode mudar o comportamento cognitivo. Pode-se inferir que, devido aos fatores biológicos não apresentarem poder para interferir significativamente no presente momento, pois o risco para o desenvolvimento não é constituído pela prematuridade por si só, não houve diferença significativa entre os participantes da pesquisa no presente estudo ${ }^{10,11,12}$

Ao analisar a variável socioeconômica dos participantes, viu-se que entre as famílias das crianças dos dois grupos não houve diferença estatística relevante, considerando que ambos os grupos são pertencentes a classes econômicas vulneráveis. De acordo com a literatura, o fator econômico interfere no bem-estar emocional dos responsáveis, influenciando no crescimento e desenvolvimento infantil adequado. Em estudos com pré-escolares houve o indício de que o nível socioeconômico mais baixo pode prejudicar o desenvolvimento cognitivo de crianças $8,13,14$

Achados na literatura sugerem que o desenvolvimento cognitivo na infância depende de condições ambientais, implicando que, ao inserir a criança em ambientes mais favoráveis economicamente, pode-se promover uma melhora em seu desenvolvimento. Além de que, fatores associados à pobreza, tais como bens de consumo, serviços $\mathrm{e}$ estímulos psicossociais insuficientes, têm sido considerados fatores de risco para o desenvolvimento infantil $^{8}$. Vale ressaltar que, mesmo não havendo fatores biológicos desfavoráveis para as crianças ao nascerem, AT avaliadas no presente estudo, um ambiente inadequado poderá trazer condições negativas para o desenvolvimento e, desta forma, interferir nesse processo.

Em um estudo em que foram avaliados, em 92 crianças com idades entre 24 e 36 meses, o desenvolvimento cognitivo e linguístico, qualidade dos ambientes educacionais, ambiente doméstico e qualidade do bairro, houve prevalência de resultados abaixo da média para o desenvolvimento cognitivo e linguístico dos participantes, os quais apresentaram resultados negativos com relação aos fatores ambientais e socioeconômicos. Além disso, as variáveis ambientais, como vizinhança, casa, e escolas de educação infantil, mostraram ter maior associação com o desenvolvimento destas crianças ${ }^{8}$.

O RNPT encontra-se em uma situação de risco para alteração ou atraso no desenvolvimento global. Por esta razão, preconiza-se o atendimento diferenciado a esse grupo de crianças. Para tanto, recomenda-se a prioridade da assistência e a comunicação entre os diversos níveis de assistência, assim como orientações à família e à mãe, mais especificamente. ${ }^{15}$ Devido a esta prioridade dada aos familiares de crianças do grupo PT, estes podem ter mais informações do que as famílias AT acerca da importância da estimulação e alimentação adequada (como o aleitamento materno e seus benefícios) para auxiliar o desenvolvimento global dessas crianças.

Esse fato pode ter influenciado os resultados observados nesta pesquisa, considerando que as crianças PT, apesar de possuírem a prematuridade como fator de risco no desenvolvimento, obtiveram, em sua maioria, estados nutricionais eutróficos. Pode-se inferir que a partir das orientações realizadas acerca da alimentação e estimulação adequadas oferecidas na maternidade, as famílias das crianças com maior risco para alteração preocuparam-se mais com os cuidados e as orientações recebidas anteriormente.

Ao comparar o nível de escolaridade (por anos) dos responsáveis dos dois grupos analisados nesta pesquisa, notou-se que também não houve diferença significativa entre os responsáveis do grupo 
AT e as do grupo PT. De acordo com a literatura, o nível de educação materna possui um impacto significativo no desenvolvimento neurológico de crianças, principalmente das crianças PT. O fator escolaridade de responsáveis pode ser mais importante no desenvolvimento neurológico dos recém-nascidos do que a idade gestacional e morbidades neonatais ${ }^{16}$. Entretanto, estudos apontam que o impacto positivo do nível de educação de familiares é observado de forma mais evidente com os resultados cognitivos de crianças em idade escolar ${ }^{17,18}$. Essas informações corroboram com a hipótese de que as crianças AT que apresentaram a média de escolaridade de responsáveis superior ao grupo PT, apesar de não conter diferença relevante estatisticamente, podem obter resultados cognitivos superiores mais tardiamente, ao chegarem à fase escolar.

Os resultados do presente estudo não corroboraram outros, os quais indicam que crianças nascidas AT apresentam melhor desenvolvimento do que as crianças nascidas prematuramente, considerando-as com menores habilidades cognitivas. Além disso, esses autores afirmam que as alterações presentes em crianças PT podem ser detectadas antes da idade escolar, o que não ocorreu diante da amostra analisada na presente pesquisa ${ }^{4,19}$.

Os resultados aqui analisados indicam a probabilidade de que as crianças AT apresentem melhor desenvolvimento cognitivo com o aumento de sua faixa etária. Deste modo, essas crianças poderão futuramente destacar-se quando comparadas às nascidas PT. Por outro lado, as crianças de ambos os grupos que apresentaram pontuações abaixo da média deverão ser acompanhadas e estimuladas para que não haja prejuízos futuros em seu desenvolvimento $10,13,20$.

\section{Conclusão}

De acordo com os resultados apresentados, sugere-se que as crianças com indicadores de risco biológico e social sejam acompanhadas e estimuladas durante o desenvolvimento, com o objetivo de remediar qualquer dificuldade que possa surgir no processo de desenvolvimento cognitivo.

\section{Referências bibliográficas}

1. Boulhoça ACS, de Lima TH. Instrumentos usados na avaliação cognitiva no ensino fundamental: análise da produção científica. Avaliação Psicológica. 1 de octubre de 2017; 20161567-76. Disponibleen: http://www.redalyc.org/articulo. oa? $\mathrm{id}=335049854008$.

2. Oliveira LD, Flores MR, de Souza, AR. Fatores de risco psíquico ao desenvolvimento infantil: implicações para a Fonoaudiologia. Mar/Apr2012; Revista CEFAC . Vol. 14 Issue 2, p333-342. 10p. 1 Chart.

3. Magalhães LdeC, Fonseca KL, Martins LDTB, Dornelas L.deF. Desempenho de crianças pré-termo com muito baixo peso e extremo baixo peso segundo o teste Denver-II. Out./dez, 2011; Rev. Saúde matern. Infant, Recife, (4): 445-453;

4. Silveira KA, Enumo SRF. Riscos biopsicossociais para o desenvolvimento de crianças prematuras e com baixo peso. Sept./Dec. 2012, vol.22 no.53 Ribeirão Preto, Paidéia.

5. Maggi FE, Magalhães LC, Campos AF, Bouzada MCF. Preterm children have unfavorable motor, cognitive, and functional performance when compared to term children of preschool age. 2014, J Pediatr (Rio J); 90(4): 377---383

6. Eickmann SH, Emond AM, Lima M. Evaluation of child development: beyond the neuromotor aspect. 2016; J Pediatr (Rio J). 92 (3 Suppl) 1: 571- 583.

7. Silva SDSH, Filho L, Gama MEA, Lamy ZC, Pinheiro AL, SIlva DN. Instrumentos de avaliação do desenvolvimento infantil de recém-nascidos prematuros. 2011; Ver.Bras Crescimento Desenv. Hum. 21(1): 85-98.

8. da Rocha Neves K, de Souza Morais RL, Teixeira RA, Pinto PA. Growth and development and their environmental and biological determinants. 2016; J Pediatr (Rio J). 92:241---50.

9. Hadders-Algra M. General movements: a window for early identification of children at high risk for developmental disorders. 2004; J Pediatr. 145: S12---8.

10. Hadders-Algra M. Social and biological determinants of growth and development in underprivileged societies. 2016; J Pediatr (Rio J); 92:217---9.

11. Räikkönen K, Pesonen AK. Early life origins of psychological development and mental health. Scand J Psychol. 2009; 50: 583---91.

12. Soares ACC, Silva K, Zuanetti PA. Risk factors for language development associated with prematurity. Audiol Commun Res. 2017; 22: e1745

13. Electronic Document Format(ISO)

14. Silva TM, Bueno NB, Azevedo MLSG, Clemente APG, Florêncio TMMT. Desempenho cognitivo de pré-escolares com baixa estatura em tratamento de recuperação nutricional. Rev. paul. pediatr. 2018, vol.36, n.1

15. Bradley RH, Pennar A, Glick J. Home environments of infants from immigrant families in the United States: findings from the new immigrant survey. Infant Ment Health J. 2014; 35: 565-79.

16. Silva, JCM. Assistência ao Recém Nascido Pré Termo de acordo com estudos brasileiros. Brasília: Monografia (PósGraduação em Fisioterapia em terapia intensiva) - Universidade Católica de Brasília, 2015. $11 \mathrm{f}$. 
17. Patra K, Greene MM , Patel LA, Meier P. O nível de educação materna prevê resultados cognitivos, de linguagem e de motor em lactentes prematuros no segundo ano da vida. Am J Perinatol . Manuscrito do autor; Publicado em forma editada final como: Am J Perinatol. 2016 Jul; 33 (8): 738-744.disponível em PMC 20161 de julho.

18. Voss W, Jungmann T, Wachtendorf M, Neubauer AP. Longterm cognitive outcomes of extremely low-birth-weight infants: the influence of the maternal educational background. 2012; Acta Paediatr. 101(6): 569-573

19. Rodrigues MC, Mello RR, Silva KS, Carvalho ML. Risk factors for cognitive impairment in school-age children born preterm: application of a hierarchical model. . 2012; ArqNeuropsiquiatr70(8): 583-589.
20. Eliane F. Maggi, Lívia C. Magalhães, Alexandre F. Campos, Maria Cândida F. Bouzada. Crianças pré-termo apresentam desempenho motor, cognitivo e funcional desfavorável em relação a neonatos a termo em idade pré-escolar. July/Aug. 2014http; J. Pediatr. (Rio J.) vol.90 no.4 Porto Alegre ://dx.doi. org/10.1016/j.jped.2013.10.005

21. Blauw-Hospers CH, Hadders-Algra M. A systematic review of the effects of early intervention on motor development. Dev Med Child Neurol. 2005; 47: 421-32. APUD SILVA TAÍSE: http://www.scielo.br/scielo.php?script=sci_arttext\&pid=S010305822018000100039\&lang=pt\#B16 